Crime, Histoire \& Sociétés / Crime, History \& Societies

Vol. 21, n² | 2017

L'histoire de la criminalité et de la justice pénale : propositions de recherche pour le $21^{\mathrm{e}}$ siècle

\title{
Crime et justice au prisme de l'histoire visuelle et matérielle
}

Xavier Rousseaux

\section{(2) OpenEdition}

Journals

Édition électronique

URL : http://journals.openedition.org/chs/2010

DOI : $10.4000 /$ chs. 2010

ISSN : 1663-4837

Éditeur

Librairie Droz

Édition imprimée

Date de publication : 31 décembre 2017

Pagination : 321-334

ISSN : $1422-0857$

Référence électronique

Xavier Rousseaux, "Crime et justice au prisme de l'histoire visuelle et matérielle », Crime, Histoire \&

Sociétés / Crime, History \& Societies [En ligne], Vol. 21, n² | 2017, mis en ligne le 19 juillet 2020,

consulté le 13 janvier 2021. URL : http://journals.openedition.org/chs/2010 ; DOI : https://doi.org/

$10.4000 /$ chs. 2010 


\title{
Crime et justice au prisme de l'histoire visuelle et matérielle
}

\author{
Xavier Rousseaux \\ «Justice [...] should manifestly and undoubtedly be seen to be done» ${ }^{1}$
}

Dorté par la révolution numérique, le statut des traces matérielles et culturelles du crime et de la justice a changé. Plutôt que de servir d'illustration à des discours écrits savants ou d'objets de collection pour amateurs fortunés, objets et monuments deviennent des pièces centrales pour comprendre comment les sociétés construisent des appareils de résolution des conflits ${ }^{2}$. Les portails consacrés à l'histoire criminelle, comme l'Old Bailey, Digital Panopticon ou Criminocorpus mettent en évidence l'intérêt pour une histoire en trois dimensions du crime et de la justice. Les humanités numériques invitent le chercheur en histoire criminelle à réinterroger des traditions de recherche consacrées à l'analyse des traces du crime et de la justice.

On peut considérer que l'approche matérielle et visuelle de la justice repose sur une double tradition ${ }^{3}$. D'une part, l'étude des représentations visuelles de la justice et du crime (peinture, sculpture, architecture) s'inscrit dans le développement de l'histoire de l'art, en particulier de l'iconologie ${ }^{4}$. D'autre part, la recherche d'une culture juridique dépassant le droit écrit, constituée de gestes, discours, objets et lieux spécifiques, s'enracine dans une archéologie particulièrement développée dans la tradition du droit germanique ${ }^{5}$. Iconologie et archéologie juridique se déploient en un double mouvement. Le premier s'attache à une histoire des représentations de la justice qui s'inscrit dans la culture artistique médiévale et classique des allégories morales. Le plus célèbre exemple en est la représentation de Justitia, censée refléter un «idéal» de la justice humaine, miroir de la justice divine ${ }^{6}$. En contrepoint, s'est développé, fin $\mathrm{XVIII}-\mathrm{XIX}$ siècle, un romantisme noir, fondé sur la fascination

1 [it is not merely of some importance but is of fundamental importance that] justice should not only be done, but should manifestly and undoubtedly be seen to be done. Lord Heward; R v Sussex Justices, ex parte McCarthy (1924) 1 KB 256, (1923) All ER Rep 233.

2 Resnik et Curtis (2011).

3 Kocher (1992); De Win (1992); Jacob (1994).

4 Panofsky (1987).

$5 \quad$ Amira et Schwerin (1943); Schild (1980, 1995, 1997, 2010); Maisel (1992); DeWin (1992); Carlen (1995, 2002); Auler (2008, 2010, 2012).

6 Kissel (1984); Jacob (1996); Sbriccoli (2005); Coll. (2016).

Crime, Histoire \& Sociétés / Crime, History \& Societies 2017, vol. 21, n² 2, pp. 321-334. 
d'objets censés rendre concrètes les pratiques pseudo-médiévales de la torture ${ }^{7}$, de l'exécution capitale et des enfermements obscurs ${ }^{8}$. Particulièrement vivace dans l'espace de l'Europe du Nord, l'archéologie juridique a connu un déclin après la Seconde Guerre mondiale en raison de ses liaisons sulfureuses avec les sciences nazies. Toujours cité, le manuel fondateur reste l'Einführung in die Rechtsarchäologie publié par von Schwerin sous le patronage de Karl von Amira9

Depuis les années 1960, l'essor d'une histoire sociale des pratiques criminelles et pénales, fondée sur l'exploitation des archives de la résolution des conflits renforce l'intérêt pour ces représentations allégoriques ou ces traces matérielles, réintégrées dans un contexte sociétal à la fois quantitatif et qualitatif. Depuis les années 1990, diverses approches sociales et culturelles combinent étude des représentations, investigations sur les expériences sensibles et plongées dans les émotions de communautés disparues et renouvellent ainsi l'intérêt pour les images et les objets ${ }^{10}$.

\section{PERCEPTIONS ET EXPÉRIENCES: CULTURES VISUELLES ET RITUELS ANTHROPOLOGIQUES}

Une telle histoire sensible de la justice criminelle se déploie autour de deux domaines d'expériences: des cultures visuelles faites de représentations iconiques fixes ou animées, en deux ou trois dimensions (images, objets, édifices), d'une part; et des rituels anthropologiques de la justice, qui s'y déroulent, d'autre part. Ces deux domaines s'imbriquent, comme les sources textuelles, visuelles ou matérielles qui en permettent la reconstruction.

Les travaux récents s'inscrivent dans une réflexion sur le pouvoir de l'image comme matrice des débats de société contemporains ${ }^{11}$. Aux recherches sur l'univers culturel des producteurs artistiques, s'ajoute une histoire fine des techniques de communication, ainsi que des enquêtes sur la réception et les représentations des publics. Au-delà des descriptions, les liens restent cependant encore complexes et difficiles à établir dans l'interaction entre productions culturelles, contextes de réception et recompositions par les acteurs.

Ainsi, l'étude des représentations visuelles s'efforce de relier l'histoire traditionnelle du droit à la plus récente histoire sociale des pratiques juridiques et judiciaires, sur base du questionnaire de l'historien des cultures visuelles ${ }^{12}$. Dans cette perspective, l'artefact offre une voie de compréhension du langage utilisé par l'artiste ou l'artisan pour communiquer avec des publics spécifiques. La manière dont les représentations collectives ont été fabriquées à un moment donné permet d'accéder aux valeurs partagées, aux priorités communes à un groupe ou aux élites d'une communauté. L'objet d'art contribue à transformer l'ancienne «histoire des mentalités» en une histoire des représentations collectives. L'interprétation des

\footnotetext{
Held (1983).
}

Coll. (1981).

$9 \quad$ Amira et Schwerin (1943); Simon (1991); Rückert et Willoweit (1995); Chapoutot (2014).

10 Rosenwein (1989, 2006); Cohen (2010); Chauvaud (2010); Hofman (2017).

11 Boehm (1994); Boehm et Mitchell (2009); Wirth (2013); Mitchell (2015).

12 Pfleister et Schild (1988); Wagner et Sherwin (2013). 
représentations reste cependant une opération complexe qui implique de considérer les conditions de production, la spécificité du producteur de l'œuvre, la matérialité de celle-ci et, avec davantage de difficultés, la réception de la communauté de spectateurs, voire de l'individu. De plus, dans les sociétés où la maîtrise des pratiques de l'écrit demeure la prérogative d'une minorité, les gestes, les images ou les objets jouent un rôle majeur dans le pouvoir de communiquer les normes ou l'identité collective $^{13}$.

Quant au tournant spatial dans les sciences sociales, il est également connecté au renouvellement des notions anthropologiques de perception, de communication et d'expérience émotionnelle ${ }^{14}$. Les développements récents en neurosciences amènent à repenser la question du rapport corps/esprit. Individuelles ou collectives, les représentations ne peuvent pas être séparées des expériences corporelles vécues par les groupes ou les individus. Or, ces expériences peuvent être canalisées, voire instrumentalisées par des pouvoirs. Ainsi, la gestion de l'espace dans les rituels publics est un moyen pour les autorités de créer un «consensus», en particulier dans les communautés d'interconnaissance. Il n'en reste pas moins que la perception concrète de cet espace par des groupes et des individus demeure une expérience spécifique, dépendant pour une part de réflexes culturels intégrés de longue date et transmis dans la communauté par la socialisation ${ }^{15}$ et, pour une autre, des sensibilités individuelles, nourries d'expériences personnelles, créant ainsi des communautés émotionnelles ${ }^{16}$.

En reliant l'étude des représentations visuelles avec l'analyse des rituels dans leur temporalité et leur spatialité, l'histoire du crime et de la justice aborde les champs de la perception, de l'expérience et de l'émotion. Appuyé sur des évolutions technologiques multipliant les traces multi sensorielles, le tournant «spatial» comme le tournant «iconique» invitent l'historien de la justice criminelle à revisiter deux problématiques: celle de la typologie des sources, et celle d'une histoire longue des cultures visuelles avec ces chantiers en cours.

\section{DOCUMENTS ET MONUMENTS: UNE TYPOLOGIE DES SOURCES}

Comme le soulignait Robert Jacob, l'étude des images de la justice met en évidence les transformations sur la longue durée des modes de représentations d'une pratique fondamentale de la société: régler les litiges ${ }^{17}$. S'appuyant sur une analyse des productions dans une vaste aire culturelle d'Europe occidentale (monde germanique, Pays-Bas, France), il évoquait trois registres de représentation, trois modes de production d'une culture visuelle des régulations sociales en Occident. La première émane des intellectuels, spécialistes de l'écrit, avec la production puis la diffusion, dès le Haut Moyen Âge de textes enluminés comme le Décret de Gratien ou le Sachsenspiegel ${ }^{18}$ évoluant avec l'imprimerie au tournant des XVe et XVI' siècles en

\footnotetext{
13 Wirth (1989); Raynaud (1990); Schmitt (1990).

14 Voir la contribution de Falk Bretschneider dans ce volume.

15 Goffman (1963).

16 Rozenwein $(1989,2006)$.

17 Jacob (1993).

18 Schmidt-Wiegand (1993).
} 
manuels de droit illustrés, liant intimement image et texte, dans un but de représenter un nouvel univers du crime, de la justice et des pouvoirs ${ }^{19}$. Un deuxième registre s'adresse à l'ensemble des groupes sociaux participant d'une vie commune dans les campagnes et les villes, il s'agit des rituels ou des performances, qui rendent le litige, son jugement et l'exécution de la décision, visibles et sensible ${ }^{20}$. En matière pénale, jouant sur les espaces, les temporalités, les vêtements, les objets, les paroles, les gestes et les textes, les liturgies d'exécution publique sont propices à la création d'un univers sensible, à la fois temporel et spatial, gestuel, visuel et textuel ${ }^{21}$. Un troisième registre met en évidence les transformations des «lieux» de justice en Occident, notamment par la couverture progressive d'édifices permanents de justice dans les paysages distinguant lieu du jugement et de la sanction. Dans l'espace du jugement, arbres et collines de justice cèdent le terrain aux salles de justice décorées, puis à la création d'immeubles spécifiques à l'exercice de la décision judiciaire: temples et palais de justice ${ }^{22}$. Dans l'espace de la sanction, où les piloris se complètent des prisons $^{23}$, apparaissent des commissariats de police et casernes de gendarmerie, qui matérialisent la réponse institutionnelle de la société au crime. À ces édifices produits par une architecture normative, celle des pouvoirs publics, il faudrait ajouter des traces plus spontanées comme les ex-voto, les croix mémorielles rappelant aux passants les homicides commis ou les mausolées improvisés après les attentats terroristes, inscrivant la mémoire douloureuse de la violence dans le paysage quotidien.

\section{UNE HISTOIRE LONGUE DES REPRÉSENTATIONS DE LA JUSTICE}

À l'instar de l'approche d'histoire sociale des archives judiciaires, les représentations du crime et de ses protagonistes ne peuvent s'appréhender qu'en liaison avec les représentations du droit et de la justice. Ces dernières renvoient à un imaginaire codifié, millénaire, lentement transformé au cours du temps, tandis que les secondes renvoient à des sensibilités plus labiles combinant représentations collectives et expériences communautaires ou individuelles particulières.

Robert Jacob a mis en évidence les deux représentations archétypiques légitimant la justice humaine. Le modèle le plus ancien qui prévaut en France et en «Allemagne » est celui de l'investiture du Christ qui confère son pouvoir au chef de l'État et au chef de l'Église. Ce modèle, qui se fixe dans le courant du XIV siècle, connaît le succès dans le monde germanique. En France à la même époque, le modèle de référence est le «Christ de Justice» représenté par une scène de crucifixion. Au XIV ${ }^{e}$ siècle, ces représentations judiciaires entrent en osmose avec les représentations religieuses, classiques depuis le $\mathrm{IX}^{\mathrm{e}}$ siècle, que sont les scènes de jugement dernier mettant en évidence le Christ-juge et l'enfer comme système pénal ${ }^{24}$. Au même moment, la

19 Becker-Moelands (1985); Prinz (2006); Raffield (2007); Dufrasne (2014, 2015); Follain et Papillard (2015).

20 Garapon (1985).

21 Franke (1985); Cohen (1989); Bastien (2006); Terpstra (2008).

22 Jacob et Jacob-Marchal (1992); Fischer-Taylor (1993); Klemmers et al. (1993); Floor (2012).

23 De Win (1996); Evans (1982).

24 Harbison (1975); Baschet (1993). 
figure du juge intègre ou prévaricateur contribue à assurer la légitimité du juge laïc, appuyée sur son serment, et autorise une sacralisation de la justice humaine ${ }^{25}$ visible dans les tableaux de justice de nombreuses villes germaniques, helvétiques ou des Pays-Bas ${ }^{26}$. Le développement des techniques imprimées popularise, à l'usage des juristes laïcs, des dessins et gravures qui distillent une vision de la justice pénale faisant la part belle aux pratiques de torture et de peines corporelles. Dans son célèbre dessin Justitia (1559), Pieter Bruegel l'ancien combine dans la même image les représentations concrètes du théâtre des supplices et l'image traditionnelle de la Justitia, vertu et pouvoir ${ }^{27}$. Par la suite, les allégories de la justice se multiplient, présentant une vertu idéalisée en tension avec les pratiques corrompues des juges terrestres.

Ce cadre de lentes transformations donne sens aux représentations jusqu'ici isolées de cette histoire des représentations ou de leur contexte judiciaire. Par exemple, le tableau de Pierre-Paul Prud'hon La Justice et la Vengeance poursuivant le Crime, destiné à orner la salle de la cour d'assises de Paris, dessine un nouveau fondement de la justice criminelle, à l'aube du XIX ${ }^{\mathrm{e}}$ siècle. Détail significatif, la vengeance est présentée comme une «agente terrible de la Justice», dont le rôle est d'appréhender le criminel et de le livrer à la justice. Désormais, dans le nouveau système punitif mis en place par la Révolution française et consolidé par l'Empire napoléonien, le code définit les crimes et les peines, le ministère public poursuit (au nom de la victime) le coupable devant les juges. Le citoyen-juré, siégeant à la cour d'assises se voit proposer une construction où la vengeance (Némésis) est devenue le bras armé de la justice (Thémis) ${ }^{28}$.

\section{UNE HISTOIRE SENSORIELLE ET SOCIALE DE LA PÉNALITÉ}

À l'autre extrémité du processus pénal, l'expression de la pénalité est considérée comme un révélateur des valeurs collectives d'une société29. Les châtiments corporels et les pratiques d'enfermement sont les peines les plus visibles dans l'espace social. Leur mode d'occupation de l'espace diffère cependant fortement dans le temps.

L'exercice public du châtiment est un «événement» particulièrement intéressant pour une approche combinant image et espace. La représentation visuelle, l'espace et les objets sont en effet au cœur du rituel et de sa mémoire. Prendre en compte la dimension spatiale et la représentation visuelle peut réconcilier les interprétations dominantes, traditionnellement fondées sur des témoignages écrits. En Occident, le développement des manifestations d'exécution publique dans l'espace de la communauté puis la tendance d'évacuation de la peine capitale de cet espace - et donc de la représentation commune - est interprétée comme un élément-clé d'un long processus de civilisation ${ }^{30}$. Dans une archéologie du pouvoir de longue durée,

\footnotetext{
25 Drüppel (1981); Coll. (2016).

26 Lederle (1937); Dupont-Bouchat et Rousseaux (1985), De Ridder (1989); Robert (1996).

27 Kissel (1984); Robert (1993); Vancauwenberghe (2003); Sbriccoli (2005).

28 Chauvaud et Vernois (2004); Coll. (2010); Gauvard et Zorzi (2015).

29 Durkheim (1899-1900).

30 Spierenburg (1984); Blok (1989); van Dülmen (1990); Gatrell (1994); Evans (1996); Bastien (2006);
} 
de nouvelles recherches mettent l'accent sur les relations entre la peine de mort et les pouvoirs locaux, mais aussi entre les rituels, les lieux et les corps ${ }^{31}$. Ainsi, un courant de l'archéologie s'est intéressée aux «ensevelissements déviants (deviant burials), dans l'Angleterre saxonne puis, dans d'autres régions de l'Europe du Nord et du centre, des fouilles ont été réalisées à l'endroit de gibets identifiés, mettant au jour des cadavres d'exécutés depuis le Haut Moyen Âge jusqu' au XIX ${ }^{\mathrm{e}}$ siècle $^{32}$. Outre les fouilles archéologiques à l'endroit des supplices, des entreprises de cartographie systématique des lieux de justice devraient être développées dans la perspective d'une archéologie du pouvoir de punir ${ }^{33}$. Les travaux sur les exécutions à l'époque médiévale et moderne se sont ainsi enrichis d'approches sur les rituels de torture ${ }^{34}$ ou de mise à mort, ont exploré la figure de l'exécuteur ${ }^{35}$, l'analyse des gestes, paroles et expressions du public, le destin des cadavres ou le sort de l'instrument de supplice ${ }^{36}$, y compris des mises à mort «populaires ${ }^{37}$. Au XIX ${ }^{\mathrm{e}}$ siècle, la guillotine focalise l'attention des savants ${ }^{38}$, mais aussi des artistes, sur le crâne du criminel exécuté. Les sources photographiques, voire cinématographiques renouvellent l'approche des scènes de justice «populaire» ou encore les exécutions publiques dans les colonies occidentales ${ }^{39}$, les lynchages dans le sud des États-Unis ${ }^{40}$, les exécutions publiques nationales ${ }^{41}$, ou en contexte de guerre, les exécutions par les tribunaux militaires, les charniers du génocide par balle, les exécutions sommaires au sortir des guerres mondiales ${ }^{42}$.

Ces enquêtes ont été particulièrement bien documentées dans le cas de peines corporelles non létales ${ }^{43}$. Les tontes de femmes en France à la fin de la Seconde Guerre mondiale ${ }^{44}$ mettent en lumière une combinaison de rituels anthropologiques structurant les rôles sexués avec des émotions collectives suscitées par la situation d'occupation et le traumatisme de la défaite de 1940. Un intérêt supplémentaire à la démarche de Virgili est d'avoir pu, sur base d'une étude systématique, donner ensuite la parole à des victimes et témoins de certaines tontes, dans un documentaire télévisue ${ }^{45}$.

L'autre forme de pénalité qui imprime sa marque dans le paysage social est la privation de liberté, considérée comme la marque pénale des sociétés modernes, de

Martschukat (2000); Friedland (2012); Ward (2015).

31 Rebecchini (2013).

32 Auler (2008, 2010, 2012); Murphy (2008); Reynolds (2009); Van der Sanden et Luning (2010). Voir aussi Redfern (2016).

33 De Win (1996); Maisel (1992); Auler (2008, 2010, 2012); Porret et al. (2012).

34 Silverman (2001).

35 Nowosadtko (1994); Stuart (2000); Klemettilä (2006).

36 Wilf (1993); Gattrell (1994); Ward (2015).

37 Corbin (1990).

38 Renneville (2000); Boariu (2015).

39 Vellut (1992).

40 Allen et al. (2012); Pfeifer (2004); Wood (2011).

${ }_{41}$ Carol $(2012,2017)$.

42 Schneidermann (2005).

43 Geltner (2014).

44 Virgili (2000).

45 Carlon (2007). 
Durkheim à Foucault ${ }^{46}$. À la différence du châtiment spectaculaire, relevant d'une logique de l'événement ${ }^{47}$, les enfermements marquent la permanence de la pénalité dans le paysage, par l'architecture de bâtiments inscrits dans les paysages urbains, puis ruraux ${ }^{48}$. De nouvelles études devraient historiciser l'enracinement progressif de cette architecture «normative» dans le paysage occidental souligné plus haut. La transition de bâtiments récupérés vers des constructions spécifiques à l'époque moderne et le monopole progressif des autorités publiques dans la construction des espaces carcéraux nourrissent un dialogue entre ces monuments publics: commissariat de police, palais de justice et prison, qui transforme la dialectique spatiale antérieure: échafaud, hôtel de ville et porte des remparts.

\section{UN CHANTIER EN COURS: HISTORICISER LES REPRÉSENTATIONS DU CRIME ET DU CRIMINEL}

Quant aux représentations du crime et de ses protagonistes, elles s'inscrivent dans une histoire culturelle, dont on peut brièvement dégager trois évolutions majeures, en lien avec la démocratisation des techniques de diffusion de l'écrit, de l'image et du son. Jusqu'au XVII siècle, une construction codée du crime et du criminel s'appuie, notamment dans l'Occident chrétien, sur les schémas religieux du martyre. Par son ambiguiité, la représentation du martyre permet au spectateur de transférer l'image de la victime sur celle du criminel suppliciée ${ }^{49}$. Le développement des techniques de l'imprimé permet au $\mathrm{XVI}^{\mathrm{e}}$ siècle l'émergence de représentations «popularisées» des auteurs du crime, à travers diverses feuilles: broadsheets et balades, occasionnels et canards, gravures et esquisses. Ces médias favorisent les représentations de profils criminels stéréotypés: hérétiques, sorcières, vagabonds, pirates, bandits, meurtriers d'enfants, au détriment du crime, lui-même difficile à représenter ${ }^{50}$. À l'âge industriel, l'essor de la presse permet de rapprocher la scène de crime des lecteurs ${ }^{51}$, tandis que la photographie focalise l'objectif sur la figure du criminel ${ }^{52}$. Au XX $\mathrm{XX}^{\mathrm{e}}$ siècle, les médias audiovisuels: cinéma, télévision, privilégient la construction de certaines formes spectaculaires comme le criminel passionnel, le meurtrier compulsif ou le crime organisés ${ }^{53}$. Enfin, des travaux devraient scruter l'émergence plus récente des représentations de la victime, amplifiées par les usages des médias contemporains ${ }^{54}$.

\footnotetext{
46 Foucault (1975).

47 Handelman (1998).

48 Spierenburg (1991); Bretschneider (2008); Geltner (2008).

49 Ortalli (1979); Edgerton (1985); Puppi (1991); Merback (1999); Groebner (2004); Mills (2005).

50 Clair (2010); Hanselaar (2017).

51 Kalifa (1995, 2005); Chauvaud (2007, 2010).

52 Regener (1999).

53 Clary et Dean (2003).

54 Tsikounas (2017).
} 


\section{CRIME ET JUSTICE COMME LIEUX DE MÉMOIRE}

Devant ce foisonnement de sources, de méthodes et de perspectives, un double défi attend l'historien de la culture visuelle et matérielle du crime et de la justice. D'une part, les modes de popularisation du passé se transforment dans une logique économique et sociale de promotion des loisirs. Littérature, émissions télévisuelles ${ }^{55}$, festivals multimédias, expositions et musées du crime et de la justice fleurissent. L'intérêt pour la littérature du «True Crime» ou la promotion du Dark Tourism, souvent fondé sur les représentations émotionnelles et influencé par des imaginaires industriels et postindustriels interroge les sciences humaines et sociales et nécessite un discours critique sur les représentations sous-jacentes aux débats d'opinion sur le crime et la justice ${ }^{56}$.

D'autre part, construire une histoire matérielle scientifique du crime et de la pénalité, dans un contexte de financement public en déclin est d'autant plus délicat que les choix d'affectation des moyens de la conservation sont sous-tendus par des idéologies nationales. À ce titre l'entreprise défendue par Jean-Claude Vimont pour l'espace français, plaidant pour une entreprise de documentation du patrimoine carcéral offre une voie exemplaire, de même que le récent «Atlas du crime à Paris $»^{57}$. D'autres phénomènes, comme la mise à disposition de l'historien des enquêtes socioanthropologiques massives réalisées dans le cadre des poursuites internationales pour crimes contre l'humanité (Nuremberg/Tokio, Rwanda, Cambodge, BosnieHerzégovine, etc. $)^{58}$, le réexamen des charniers de meurtres de masse à la lueur des progrès des sciences forensiques, voire la réouverture de cold cases grâce aux technologies nouvelles plaident pour une conservation patrimoniale des traces du crime et de la justice. Ces tendances rappellent à l'historien du droit, du crime et de la justice son rôle dans le développement d'une histoire publique. Dans ce cas son expertise des investigations en profondeur des traces sociétales du crime et de la justice peut nourrir les débats citoyens et politiques sur la signification des phénomènes criminels et de la réaction sociale au crime.

Xavier Rousseaux FRS-FNRS

Université catholique de Louvain Centre d'histoire du droit et de la justice xavier.rousseaux@uclouvain.be

\section{BIBLIOGRAPHIE}

Allen, J., Als, H., Lewis, J. et Litwack, L.F (2012) Without Sanctuary: Lynching Photography in America, Santa Fe: Twin Palms Publishers, $11^{\mathrm{e}} \mathrm{ed}$.

Amira, K. von, Schwerin, C. von (1943) Einführung in die Rechtsarchäologie, Berlin-Dahlem: Ahnenerbe-Stiftung Verlag.

Auler, J. (Ed.) (2008) Richtstättenarchäologie, Dormagen: Archaeotopos-Buchverlag, t.1.

\footnotetext{
55 Tsikounas (2017).

56 Dalton (2015); Hooper et Lennon (2016).

57 https://clamor.criminocorpus.org/ [consulté le 17 novembre 2017]; voir Kalifa et Farcy (2015).

58 Voir la contribution d'Ornella Rovetta dans ce volume.
} 
Auler, J. (Ed.) (2010) Richtstättenarchäologie, Dormagen: Archaeotopos-Buchverlag, t.2.

Auler, J. (Ed.) (2012) Richtstättenarchäologie, Dormagen: Archaeotopos-Buchverlag, t.3.

Baschet, J. (1993) Les justices de l'au-delà. Les représentations de l'enfer en France et en Italie $\left(X I I^{e}-X V^{e}\right.$ siècle), Rome: École Française de Rome.

Bastien, P. (2006) L'exécution publique à Paris au XVIII'siècle. Une histoire des rituels judiciaires, Seyssel: Champ Vallon.

Becker-Moelands, M.A. (1985) De juridische titelprent in de zeventiende eeuw: het Hof van Holland, Muiderberg: Dick Coutinho.

Blok, A. (1989) The Symbolic Vocabulary of Public Executions, in History and Power in the Study of Law: New Directions in Legal Anthropology, Ithaca NY: Cornell University Press, p. 31-54.

Boariu, D.A. (2015) La tête coupée. Clinique et peinture de la décapitation au XIXe siècle, Université de Fribourg, thèse de doctorat inédite.

Boehm, G. (1994) Was ist ein Bild?, Munich: W. Fink.

Boehm, G. et Mitchell, W.J.T. (2009) Pictorial versus Iconic Turn: Two Letters, Culture, Theory and Critique, special issue: The Pictorial Turn, 50, 2-3.

Bretschneider, F. (2008) Gefangene Gesellschaft. Eine Geschichte der Einsperrung in Sachsen vom 18. bis zum 19. Jahrhundert, Constance: UVK.

Carlen, L. (1995) Sinnenfälliges Recht: Aufsätze zur Rechtsarchäologie und rechtlichen Volkskunde, Hildesheim: Weidmann.

Carlen, L. (Ed.) (2002) Recht, Geschichte und Symbol. Aufsätze und Besprechungen, Hildesheim: Weidmann.

Carol, A. (2012) Physiologie de la veuve. Une histoire médicale de la guillotine, Seyssel: Champ Vallon.

Carol, A. (2017) Au pied de l'échafaud. Une histoire sensible de l'exécution, Paris: Belin.

Clair, J. (Ed.) (2010) Crime et châtiment, Paris: Musée d'Orsay/Gallimard.

Chapoutot, J. (2014) La loi et le sang. Penser et agir en nazi, Paris: Gallimard.

Chauvaud, F. (Ed.) (2007) Justice et déviance à l'époque contemporaine. L'imaginaire, l'enquête et le scandale, Rennes: Presses Universitaires de Rennes.

Chauvaud, F. (2010) La chair des prétoires? Histoire sensible de la cour d'assises 1881-1932, Rennes: Presses universitaires de Rennes.

Chauvaud F. et Vernois S. (2004) La Justice en images. Croquis, dessins et caricatures, Sociétés et représentations, 18.

Clary, F. et Dean J. (Eds.) (2003) Crime \& Hollywood Inc.: Le Crime organisé, à la ville et à l'écran. 1929-1951, Rouen: Presses de l'Université de Rouen.

Corbin, A. (1990) Le village des cannibales, Paris: Aubier.

Cohen, E. (1989) Symbols of Culpability and the Universal Language of Justice: The Ritual of Public Executions in Late Medieval Europe, History of European Idea, 11, p. 407-416.

Cohen, E. (2010) The Modulated Scream: Pain in Medieval Culture, Chicago: University of Chicago Press.

Coll. (1981) Criminal justice through the ages. From Divine Judgement to Modern German Legislation, Rothenburg: Criminal Museum.

Coll. (2010) Justice(s). Le Temps des medias, 15, Paris: Nouveau monde. 
Coll. (2016) The Art of Law. Three centuries of justice depicted (exh.cat.), Tielt: Lannoo.

Dalton, D. (2015) Dark Tourism and crime, Abingdon Oxon: Routledge.

De Ridder, J. (1989) Gerechtigheidstaferelen voor schepenhuizen in de Zuidelijke Nederlanden in de 14de, 15de en 16de eeuw, Bruxelles: Koninklijke Academie voor Wetenschappen, Letteren en Schone Kunsten van België.

De Win, P. (Ed.) (1992) Rechtsarcheologie en rechtsiconografie. Een kennismaking, Bruxelles: Koninklijke Academie voor Wetenschappen, Letteren en Schone Kunsten van België.

De Win, P. (1996) Inventaris van de feodale schandpalen op Belgisch grondgebied, 3 vol., Bruxelles: Koninklijke Academie voor Wetenschappen, Letteren en Schone Kunsten van België.

Drüppel, H. (1981) Iudex Civitatis. Zur Stelling des Richters in der hoc-und spätmittelalterlichen Stadt deutschen Rechts, Cologne-Vienne: Böhlau.

Dufrasne, J. (2014) Les gravures de la Praxis rerum criminalium de Jos de Damhouder: la représentation de la justice pénale du XVI siècle à partir du programme iconographique de l'œuvre (1554-1618), maîtrise en Histoire, inédite, Louvain-la-Neuve: Université catholique de Louvain.

Dufrasne, J. (2015) Les gravures dans l'œuvre du juriste Jos de Damhouder (1554-1618). Représentation de la violence dans un manuel juridique illustré, maîtrise en Histoire de l'art inédite, Louvain-la-Neuve: Université catholique de Louvain.

Dupont-Bouchat, M.S. et Rousseaux, X. (1985) Images de la justice criminelle $\left(\mathrm{XV}^{\mathrm{e}}\right.$-XVIII ${ }^{\mathrm{e}}$ siècles). Justice des hommes, justice divine, in Lamoine, G. (Ed.), Images et représentations de la justice du XVI e au XIXe siècle, Toulouse: Université de Toulouse-Le Mirail, p.37-41.

Durkheim, E. (1899-1900) Deux lois de l'évolution pénale, Année sociologique, 4, p. 65-95.

Edgerton, S. (1985) Pictures and Punishment. Art and Criminal Prosecution during the Florentine Renaissance, Ithaca/London: Cornell University Press.

Evans, R. (1982) The fabrication of virtue: English prison architecture, 1750-1840, Cambridge: Cambridge University Press.

Evans, R.J. (1996) Rituals of Retribution: Capital Punishment in Germany, 1600-1987, Oxford: Oxford University Press.

Fischer-Taylor, K. (1993) In the Theater of Criminal Justice. The Palais de Justice in Second Empire Paris, Princeton: Princeton University Press.

Floor, R. (2012) Architectuur van het Recht. Nederlandse Justitiegebouwen 1870-1914, Zutphen: De Walburg Pers.

Follain, A. et Papillard, C.A. (2015) Figures du crime et de la violence au XVI siècle: les singulières gravures insérées dans la Praxis rerum criminalium de Damhoudère, in Follain, A. (Ed.) Brutes ou braves gens? La violence et sa mesure (XVI ${ }^{e}$-XVIII ${ }^{e}$ siècle), Strasbourg: Presses Universitaires de Strasbourg, p. 37-41.

Foucault, M. (1975) Surveiller et punir. Naissance de la prison, Paris: Gallimard.

Franke, H. (1985) De dood in het leven van alledag. Rouwadvertenties en openbare strafvoltrekkingen in Nederland, La Haye: Nijgh \& Van Ditmar.

Friedland, P. (2012) Seeing Justice Done: The Age of Spectacular Capital Punishment in France, Oxford: Oxford University Press.

Garapon, A. (1985) L'âne portant des reliques. Essai sur le rituel judiciaire, Paris: Le Centurion. 
Gatrell, V. (1994) The Hanging Tree: Execution and the English People 1770-1868, Oxford: Oxford University Press.

Gauvard, C. et Zorzi, A. (Eds.) (2015) La vengeance en Europe, XII ${ }^{e}$-XVIII ${ }^{e}$ siècles, Paris: Presses Universitaires de Paris-Sorbonne.

Geltner, G. (2008) The Medieval Prison. A Social History, Princeton: Princeton University Press.

Geltner, G. (2014) Flogging Others. Corporal Punishment and Cultural Identity from Antiquity to the Present, Amsterdam: Amsterdam University Press.

Goffman, E. (1963) Behavior in Public Places; Notes on the Social Organization of Gatherings, New York: Free Press of Glencoe.

Groebner, V. (2004) Defaced: The Visual Culture of Violence in the Late Middle Ages, New York: MIT Press/Zone books.

Handelman, D. (1998) Models and Mirrors: Towards an Anthropology of Public Events, New York-Oxford: Berghahn Books.

Hanselaar, S. (2017) Édification et peinture d'histoire: des limites à la représentation du criminel et du fait divers dans le premier $\mathrm{XIX}^{\mathrm{e}}$ siècle, Tétrade. Revue du Centre de recherche en arts et esthétique, 4, p. 45-53.

Harbison, C. (1975) The Last Judgment in Sixteenth Century Northern Europe. A Study in the Relation between Art and the Reformation, New York: Garland.

Held, R. (1983) Guida bilingue alla mostra di Strumenti di Tortura dal Mediovo all'epoca industriale. A bilinguial Guide to the exhibition of Torture Instruments. From the Middle Ages to the Industrial Era, Florence: Qua d'Arno.

Hofman, E. (2017) The Tears of a Killer. Criminal Trials and Sentimentalism in the Austrian Netherlands. Low Countries Historical Review, 132, 2, p. 3-26.

Hooper G. et Lennon, J. (2016) Dark tourism: Practice and interpretation, New York: Routledge.

Jacob, R. (1993) Images de Justice. Essai sur l'iconographie judiciaire du Moyen Âge à l'âge classique, Paris: Le Léopard d'Or.

Jacob, R. et Jacob-Marchal, N. (1992) La justice en ses temples, Regards sur l'architecture judiciaire en France, Poitiers/Paris: Brissaud/Errance.

Kalifa, D. (1995) L'encre et le sang : récits de crimes et société à la Belle Époque, Paris: Fayard.

Kalifa, D. (2005) Crime et culture au XIX $X^{e}$ siècle, Paris: Perrin.

Kalifa, D. et Farcy J.C. (2015) Atlas du crime à Paris. Du Moyen Âge à nos jours, Paris: Parigramme.

Kissel, O. (1984) Die Justitia. Reflexionen über ein Symbol und seine Darstellung in der bildende Kunsten, Munich: Beck.

Klemettilä, H. (2006) Epitome of Evil. Representations of Executioners in Northern France and the Low Countries in the Late Middle Ages, Turnhout: Brepols.

Klemmers, K., Wassermann R. et Wessel T.M. (1993) Deutsche Gerichtsgebäude. Von der Dorflinde über den Justizpalast zum Haus des Rechts, Munich: Beck.

Kocher, G. (1992) Zeichen und Symbole des Rechts. Eine historische Ikonographie, Munich: C.H.Beck.

Lederle, U. (1937) Gerechtigkeitsdarstellungen in deutschen und niederländischen Rathäusern, Philippsburg: Kruse. 
Maisel, W. (1992) Rechtsarchäologie Europas, Vienne: Böhlau.

Martschukat, J. (2000) Inzseniertes Töten. Eine Geschichte der Todesstrafe vom 17. Biz zum 19. Jahrhundert. Cologne-Vienne: Bölhau.

Merback, M.B. (1999) The Thief, the Cross and the Wheel: Pain and the Spectacle of Punishment in Medieval and Renaissance Europe, Chicago: The Chicago University Press.

Mills, R. (2005) Suspended animation. Pain, pleasure and Punishment in Medieval Culture, London: Reaktion Books.

Mitchell, W.J.T. (2015) Image Science: Iconology, Visual Culture and Media Aesthetics, Chicago: University of Chicago Press.

Murphy, E.M. (2008) Deviant Burial in the Archaeological Record, Oxford: Oxbow Books.

Nowosadtko, J. (1994) Scharfrichter und Abdecker. Der Alltag zweier , unehrlicher Berufe “ in der Frühen Neuzeit, Paderborn: Schöningh.

Ortalli, G. (1979) Pingatur in palatio. La pittura infamante nei secoli XIII-XVI, Rome: Jouvence.

Panofsky, E. (1987) Essais d'iconologie. Thèmes humanistes dans l'art de la Renaissance, Paris: Gallimard.

Pfeifer, M.J. (2004) Rough Justice: Lynching and American Society, 1874-1947, Urbana: University of Illinois Press.

Pfleister, W. et Schild, W. (Eds.) (1988) Recht und Gerechtigkeit im Spiegel der europaïschen Kunst, Cologne: DuMont.

Porret, M., Fontana, V. et Maugué, L. (Eds.) (2012) Bois, fers et papiers de justice. Histoire matérielle du droit de punir, Genève: Georg.

Prinz, F. (2006) Der Bildgebrauch in gedruckten Rechtsbüchern des 15. bis zum Ausgang des 18. Jahrhunderts, Münster: LIT Verlag.

Puppi, L. (1991) Les supplices dans l'art. Cérémonial des exécutions capitales et iconographie du martyre du XII ${ }^{e}$ au XIX ${ }^{e}$ siècle, Paris: Larousse.

Raffield, P. (2007) Images and Cultures of Law in Early Modern England. Justice and Political Power, 1558-1660, Cambridge: Cambridge University Press.

Raynaud, C. (1990) La violence au Moyen Âge (XIII -XV ${ }^{e}$ s.) d'après les livres d'histoire en français, Paris: Le Léopard d'Or.

Rebecchini, G. (2013) Rituals of Justice and the Construction of Space in Sixteenth-Century Rome, I Tatti Studies in the Italian Renaissance, 16, 1-2, p. 153-179.

Redfern, R. (2016) Injury and trauma in Bioarcheology. Interpreting violence in past lives, Cambridge: Cambridge University Press.

Regener, S. (1999) Fotografische Erfassung : zur Geschichte medialer Konstruktionen des Kriminellen, Munich: Fink.

Renneville, M. (2000) Le langage des crânes. Une histoire de la phrénologie, Paris: Institut d'édition Sanofi-Interlabo.

Resnik, J. et Curtis D. (2011) Representing justice: invention, controversy and rights in citystates and democratic courtrooms, New Haven-London: Yale University Press.

Reynolds, A. (2009) Anglo-Saxon Deviant Burial Customs, Oxford: Oxford University Press.

Robert, C-N. (1993) Une allégorie parfaite. La Justice: vertu, courtisane et bourreau, Genève: Georg.

Robert, C.-N. (1996) La justice dans ses décors (XVe-XVI siècles), Genève: Droz. 
Rozenwein, B. (1989) Anger's Past: The social uses of an Emotion in the Middle Ages, Ithaca N.Y.: Cornell University Press.

Rozenwein, B. (Ed.) (2006) Emotional Communities in the Early Middle Ages, Ithaca N.Y.: Cornell University Press.

Rückert, J. et Willoweit, D. (1995) Die Deutsche Rechtsgeschichte in der NS-Zeit: ihre Vorgeschichte und ihre Nachwirkungen, Mohr: Siebeck.

Sbriccoli, M. (2005) La triade, le bandeau, le genou droit et procès pénal. Les allégories de la Justice du Moyen Âge à l'âge moderne, Crime, Histoire \& Sociétés, 9-1, p. 33-78.

Schild, W. (1980) Alte Gerichtsbarkeit - Vom Gottesurteil bis zum Beginn der modernen Rechtsprechung, Munich: Callwey.

Schild, W. (1995) Bilder von Recht und Gerechtigkeit, Cologne: DuMont.

Schild, W. (1997) Die Geschichte der Gerichtsbarkeit Vom Gottesurteil bis zum Beginn der modernen Rechtssprechung, Hambourg: Nikol Verlag.

Schild, W. (2010) Folter, Pranger, Scheiterhaufen. Rechtssprechung im Mittelalter, Munich: Bassermann Verlag.

Schmidt-Wiegand, R. (Ed.) (1993) Eike von Repgow. Sachsenspiegel. Die Wolfenbütteler Bildhandschrift, 2 vol., Berlin: Akademie Verlag.

Schmitt, J-C. (1990) La raison des gestes dans l'Occident médiéval, Paris: Gallimard.

Silverman, L. (2001) Tortured Subjects: Pain, Truth, and the Body in Early Modern France, Chicago: The University of Chicago Press.

Simon, W. (1991) Claudius Freiherr von Schwerin. Rechtshistoriker während dreier Epochen deutscher Geschichte. Francfort/Main: Peter Lang.

Spierenburg, P. (1984) The Spectacle of Suffering: Executions and the Evolution of Repression from a Preindustrial Metropolis to the European Experience, Cambridge: Cambridge University Press.

Spierenburg, P. (1991) The Prison Experience: Disciplinary Institutions and their Inmates in Early Modern Europe, New Brunswick: Rutgers.

Stuart, K. (2000) Defiled outcasts. Honor and ritual pollution in Early Modern Germany. Cambridge: Cambridge University Press.

Terpstra, N. (2008) The Art of Executing Well. Rituals of Execution in Renaissance Italy, Kirksville: Truman State University Press.

Van Der Sanden W., Luning H. (Eds.) (2010) Over galg en rad. Executieplaatsen in Drenthe, Zwolle: Waanders.

Tsikounas, M. (2017) La Caméra explore le crime. Les Causes célèbres du XIXé siècle à la télévision, Rennes: Presses Universitaires de Rennes.

van Dülmen, R. (1990) Theatre of Horror: Crime and Punishment in Early Modern Germany, Cambridge: Polity Press.

Vancauwenberghe, A. (2003) Le dessin Justicia de Pierre Bruegel l'Ancien (1559). Revue des Archéologues, historiens d'art et musicologues de l'UCL, 1, p.31-45.

Vellut, J-L. (1992) Une exécution publique à Elisabethville (20 septembre 1922). Notes sur les pratiques de la peine capitale dans l'histoire coloniale du Congo, in Jewsiewicki, B. (Ed.), Art pictural zaïrois, Québec: Septentrion, p. 171-122.

Virgili, F. (2000) La France virile. Des femmes tondues la Libération, Paris: Payot.

Wagner, A., Sherwin, R.K. (Ed.) (2013) Law, Culture and Visual Studies, Berlin: Springer. 
Ward, R. (2015) A Global History of Execution and the Criminal Corpse, Basingstoke: Palgrave. Wilf, S. (1993) Imagining Justice: Aesthetics and Public Executions in Late Eighteenth-Century England, Yale Journal of Law \& the Humanities, 5-1, p. 51-78.

Wirth, J. (1989) L’image médiévale. Naissance et développements (VI ${ }^{e}-X V^{e}$ siècles), Paris: Méridiens-Klinksieck.

Wirth, J. (2013) Qu'est-ce qu'une image?, Genève: Droz.

Wood, A.L. (2011) Lynching and Spectacle: Witnessing Racial Violence in America. 18901940, Chapel Hill: University of North Carolina Press.

\section{Sitographie}

http://www.kriminalmuseum.eu/museum-shop/?lang=en

https://clamor.criminocorpus.org/

museolombroso.unito.it/

https://www.oldbaileyonline.org/

https://www.digitalpanopticon.org/

http://www.digithemis.be/

\section{Films documentaires}

Schneidermann, D. (2005) Jour de pendaison au village, documentaire 52 minutes, Riff International Production, avec la participation de France 5 et du CNC.

Carlon, J.-P. (2007) Tondues en 44, documentaire 52 minutes, France Télévisions. 